\title{
Labyrinthe
}

23 | 2006 (1)

Quatre chercheurs de l'étranger

\section{Danielle Allen : la multiplicité du positif}

\section{Laurent Dubreuil}

\section{OpenEdition}

\section{Journals}

Édition électronique

URL : http://journals.openedition.org/labyrinthe/1158

DOI : $10.4000 /$ labyrinthe. 1158

ISSN : 1950-6031

Éditeur

Hermann

Édition imprimée

Date de publication : 15 janvier 2006

Pagination : 41-45

ISBN : 2-9526131-0-9

\section{Référence électronique}

Laurent Dubreuil, « Danielle Allen : la multiplicité du positif », Labyrinthe [En ligne], 23 | 2006 (1), mis en ligne le 23 juillet 2008, consulté le 19 avril 2019. URL : http://journals.openedition.org/labyrinthe/1158 ; DOI : 10.4000/labyrinthe.1158 


\section{Danielle Allen : la multiplicité du positif}

Laurent DUBREUIL

Danielle S. Allen est à la recherche du multiple. Talking to Strangers, son dernier ouvrage ${ }^{1}$, formule l'hypothèse d'une société accueillant la diversité, sans la conversion unitaire des différences ni l'indifférence entre cultures. Dans la conduite de sa propre action intellectuelle, Allen se risque aussi à la pluralité. Les textes grecs, la théorie politique, les recueils de lois, la poésie américaine ou les photographies documentaires donnent ensemble l'occasion à Danielle Allen de mener une recherche qui se prive de la spécialisation, mais pas de la compétence. Se lit chaque fois une exigence d'éclectisme (choix et diversité) pour la démonstration.

La formation intellectuelle d'Allen est exemplaire de cette curiosité. Après des études à Princeton, où elle hésite entre études anciennes et théorie politique, Danielle Allen finit par soutenir deux doctorats, l'un en grec à Cambridge (1996), l'autre au Department of Government de Harvard (2001). La fonction professorale qu'elle détient désormais à l'université de Chicago rend compte de l'étendue de ses intérêts, à la jonction des études classiques, des sciences politiques et sociales.

Assurément, l'œuvre d'Allen est pluridisciplinaire. Son premier livre, The World of Prometheus ${ }^{2}$, envisage la réalité sociale et politique d'Athènes selon un large spectre, des inscriptions aux tragédies, des discours oratoires aux traités philosophiques. Le problème de la punition est central, et il permet de parcourir des données textuelles et historiques discontinues. La méthode est sans doute marquée par les recherches de Foucault: l'interrogation de la discipline comme châtiment autorise le recours à plusieurs disciplines du savoir. Un souci comparable justifie

\footnotetext{
1. Talking to Strangers. Anxieties of Citizenship since Brown v. Board of Education, Chicago, The University of Chicago Press, Chicago et Londres, 2004, 232 p.

2. The World of Prometheus. The Politics of Punishing in Democratic Athens, Princeton, Princeton University Press, 2000.
} 
d'autres essais, hors du domaine grec, comme cet article sur le bruit de la machine torturante dans La Colonie pénitentiaire de Kafka ${ }^{3}$.

La régulière échappée vers les littératures européennes et américaine du dernier siècle n'empêche pas Danielle Allen de revenir aux Grecs. Pas de retour cependant. Il n'y a point chez Allen, je crois, l'envie de restituer un sens plein (oublié, déformé), ni le seul souhait de rendre sensible l'altérité du passé. Les Grecs valent pour ce qu'ils ont encore à délivrer, si l'on prend la peine de les écouter - et de les confronter à l'époque depuis laquelle nous les lisons. Ceux qui parlent de la-pensée-occidentale auraient toujours intérêt à voir s'il n'existe pas des pensées rebelles à la tradition, même quand celle-ci prétend s'arroger celles-là. La lecture à contretemps des auteurs anciens ne se réduit pas à une instrumentalisation. Plutôt, quand Talking to Strangers s'achève par Aristote, le Philosophe est sollicité pour l'étrangeté de son propos; et une autre manière de penser la relation politique se reformerait. Ou encore, le prochain ouvrage d'Allen, sur l'autorité des voix et les voix d'autorité se relie à l'obligation pour les démocraties de composer avec la menace et la terreur ${ }^{4}$.

Oui, les temps éloignés, les paroles lointaines peuvent se relier. Danielle Allen aime réunir les séparés. Ce geste est délicat, d'autant que l'auteur se défie des grandes synthèses. Allen reconnecte; ou, si l'on craint les métaphores cybernétiques et les empreintes deleuziennes, disons qu'elle renoue. Dans cette réflexion, la séparation entre les branches de la science n'est pas prise pour un absolu. Les écarts de contextes historiques et sociaux n'interdisent pas la comparaison, ou, mieux, le rapprochement. L'élaboration de modèles et d'arguments accompagne la participation à la vie collective. Il faut le noter, Allen croit en l'effectivité sociale des sciences humaines; comme dans la capacité des scholars à intervenir au sein de la vie publique autrement qu'en experts dispensateurs de la vérités. Je parlais en ce sens, tout à l'heure, d'action intellectuelle. Danielle Allen a accepté des fonctions administratives importantes à l'université de Chicago, celles de Dean pour la division des Humanités, afin de contribuer autrement à l'élaboration de

3. Danielle S. Allen, «Sounding Silence: in Kafka's "In the Penal Colony"», dans Modernism/ Modernity, vol. VIII, $\mathrm{n}^{\circ}$ 2, 2001.

4. On trouvera une première formulation de ce débat dans l'article de Danielle S. Allen, « Changing the Authoritative Voice: Lycurgus' Against Leocrates », dans Classical Antiquity, avril 2000.

5. Allen est d'ailleurs très critique envers les thèses de Jürgen Habermas (Talking to Strangers, ch. 5). 
la politique « académique ». Cette jeune femme noire a pris position, à de nombreuses reprises, pour un urbanisme contrant la ségrégation spatiale et raciale. Et ce professeur enseigne également aux adultes les plus pauvres, qui sont en marge de l'enseignement supérieur ${ }^{6}$.

De telles pratiques illustrent l'un des arguments de Talking to Strangers, dont sont issus les extraits traduits ici pour la première fois en français. La démocratie vit dans la citoyenneté, et les individus, les groupes, par leurs propres motifs et comportements, informent la vie politique. Pour Allen, les «démocraties occidentales», malgré leurs crises ou tensions, ne sont pas des lieux de confiscation définitive de la liberté par le pouvoir (de classe, de race, d'État). Les citoyens, estime l'auteur, ont possibilité de transformer l'usage, ou les «mauvaises habitudes ${ }^{7}$ », par leurs actes - où entre la catégorie du sacrifice délibéré.

Le livre commence par la riche évocation de l'affaire de Little Rock, où, en 1957, l'adolescente noire Elizabeth Eckford voulut aller au lycée en application de la décision de la Cour suprême contre l'apartheid scolaire aux États-Unis ${ }^{8}$. Le jugement de la Cour et la volonté d'Eckford sont aux yeux d'Allen un authentique moment de refondation de la Constitution américaine. Cette sorte de scène primitive, et les commentaires qu'elle avait déclenchés à son époque, configurent chez Allen un espace de combat politique pour la démocratisation de la démocratie. Convoquant des penseurs modernes de la res publica (Hobbes, Arendt, Habermas), Allen établit progressivement un modèle alternatif à l'unité organique du corps politique. La xénophilie est ainsi posée en principe vertueux. Outre l'exemple actif d'Elizabeth Eckford et la sollicitation d'Aristote (essentiellement pour la Rhétorique et l'Éthique à Nicomaque), Allen renvoie continûment à Invisible Man de Ralph Ellison, que je tiens volontiers pour le meilleur Bildungsroman de l'après-guerre. Ellison - l'écrivain et l'essayiste -, Eckford - l'activiste et la simple lycéenne sont deux guides afro-américains pour Danielle Allen dans sa reformulation d'une théorie pratique du citoyen américain aujourd'hui. Les pages

\footnotetext{
6. Sur le cadre de ce programme pédagogique intitulé The Odyssey Project, voir http://www.prairie.org/index.cfm/fuseaction/dir_programs.prog_detail/object_id/0ce31632-4076-4ce1-b839bc9a63e67e5a/TheOdysseyProject.cfm.

7. «Pourquoi nous avons de mauvaises habitudes» est le titre de la deuxième partie de Talking to Strangers.

8. Le cas juridique est désigné, selon l'usage de la jurisprudence américaine, par les noms des parties impliquées dans le procès, en l'occurrence, Brown v. Board of Education (sentence rendue par la Cour suprême en 1954).
} 


\section{Labyrinthe, $n^{\circ} 23$}

que nous donnons à lire rendent sensible une part du trajet conceptuel, depuis un Hobbes revisité jusqu'aux propositions, inspirées d'Aristote, qui servent à la reconstruction de la vie collective.

Les lecteurs admiratifs de la radicalité politique à la française pourront s'étonner que Danielle Allen accorde une telle importance à la confiance. Car si le xénophile doit postuler sa propre «vulnérabilité», ce n'est pas d'abord en vertu d'une morale inconditionnelle, mais plutôt parce qu'il croit à la démocratie (et en sa perfectibilité). Le cadre d'exercice du pouvoir n'est pas fondamentalement remis en cause par Allen. L'auteur fait parfois parler une voix, qui lui reproche sa propension à « composer $^{9} »$. Allen fait ainsi accueil à une position divergente, et, de la sorte, conforte sa propre interprétation du multiple. Elle s'oppose avec la plus grande netteté à la pseudo-révolution des néo-conservateurs américains. Elle ne rejoint pas non plus le néo-conformisme européen qui reproduit le vieil unitarisme majoritaire en l'habillant de couleurs. Mais, à l'évidence, Allen se veut réformiste. Dans cette même direction, nous dit-elle, il y a mieux. Allen s'attache longuement aux imperfections, dans la deuxième partie de son livre. Il n'y est toutefois pas fait mention de la défectivité du réel, de sa pensée, de sa mise en ordre social. Partant, le manque, pour elle, n'est pas plus une négativité constitutive qu'une défaillance accidentelle.

Danielle Allen vise une multiplicité du positif, qu'exprime la réflexivité active des sujets vivant dans la Cité. La démocratie est alors nécessaire. Elle est déterminée par l'élaboration historique d'une collectivité au contact d'autres groupes, d'autres mondes. Elle existe par sa possibilité d'hébergement du contradictoire. $L a$ démocratie demeure dans tous les cas. La parole de Danielle Allen est structurellement en phase avec cette analyse. Pluridisciplinaire, sa recherche est aussi métadisciplinaire, dans la mesure où elle passe les frontières pour énoncer un discours capable d'assurer la validité de sa pluralité épistémique ${ }^{10}$. Ici, l'intersection des savoirs n'est pas une fin en soi, et l'indiscipline n'est pas le souci premier.

L'oubli volontaire du défectif tient une place cruciale dans les analyses d'Allen. Il n'est pas si certain, par exemple, que le protagoniste

\footnotetext{
9. Par exemple Talking to Strangers, p. 118, 159.

10. L'Université en général, et celle de Chicago en particulier, est un acteur de la réflexion d'Allen (voir entre autres sa conclusion).
} 
d'Invisible Man (je ne dis pas Ralph Ellison) «aspire à la fraternité et propose des techniques de prise en charge de l'intrication du monde et de la réciprocité entre citoyens ${ }^{11} \gg$. Ce Je invisible qui termine son manuscrit dans un sous-sol illuminé par des dizaines d'ampoules électriques n'est-il pas retranché du monde auquel il s'adresse ? La douloureuse obligation de réforme est peut-être portée jusqu'à nous par la tension entre l'aspiration individuelle et les déboires accumulés; mais le personnage d'Ellison, auteur de ses confessions, nous propose-t-il quelque chose? Le texte littéraire pourrait tout autant nous inciter à souligner l'impossibilité de toute conduite politique (représentée par les hypostases successives du narrateur: le jeune étudiant idéaliste de black college, l'ouvrier, le porte-parole politique, le révolutionnaire...).

La proposition désigne le rétablissement du reste positif et héroïque qui était à l'œuvre jusque dans le retrait. Et elle est la signature de Danielle Allen. L'amitié politique, cette amicalité réinventée d'après Aristote, se vit dans un accompagnement et un dialogue. Je ne sais si la xénophilie est assez puissante pour modifier l'exercice politique, j'en douterais plutôt, mais je veux croire à tout le moins qu'un espace d'échange démocratisé puisse s'ouvrir à partir d'un livre comme Talking to Strangers. À cette promesse en plus des autres, le lecteur est ici rendu.

11. Talking to Strangers, p. 118. 